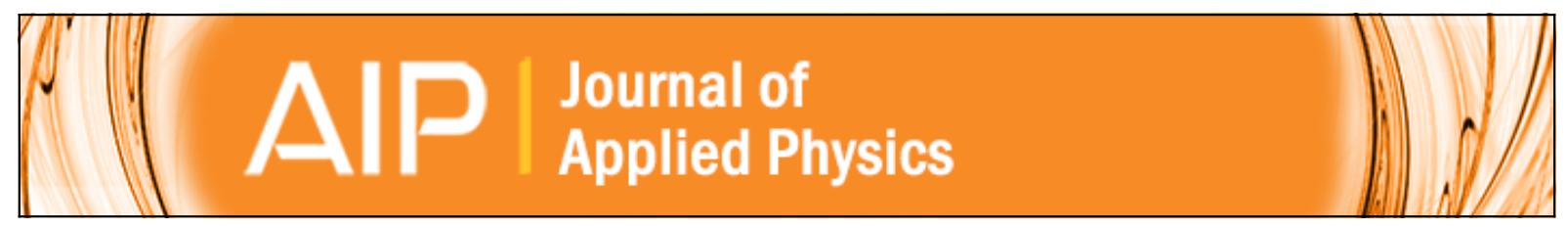

Electrically detected and conventional magnetic resonance investigation of surface and bulk states in polyaniline thin films

Fernando A. Castro and Carlos F. O. Graeff

Citation: Journal of Applied Physics 101, 083903 (2007); doi: 10.1063/1.2719007

View online: http://dx.doi.org/10.1063/1.2719007

View Table of Contents: http://scitation.aip.org/content/aip/journal/jap/101/8?ver=pdfcov

Published by the AIP Publishing 


\title{
Electrically detected and conventional magnetic resonance investigation of surface and bulk states in polyaniline thin films
}

\author{
Fernando A. Castro \\ Departamento de Física e Matemática, FFCLRP, Universidade de São Paulo, Av. Bandeirantes 3900, \\ 14040-901 Ribeirão Preto, SP, Brazil \\ Carlos F. O. Graeff \\ Departamento de Física, FC, UNESP, Av. Luiz Edmundo Carrijo Coube, 14-01, 17033-360 Bauru-SP, \\ Brazil
}

(Received 31 October 2006; accepted 21 February 2007; published online 25 April 2007)

\begin{abstract}
Electrically detected magnetic resonance (EDMR) and electron paramagnetic resonance (EPR) were used to investigate emeraldine base polyaniline films. The magnetic susceptibility presented a Curie (localized spins) - Pauli (delocalized spins) transition at $240 \mathrm{~K}$, when we also observed a transition in the dependence of the $g$ factor with temperature $(T)$. Peak-to-peak linewidth decreases with increasing temperature, reflecting that motional narrowing limits the hyperfine and dipolar broadening in this polymer. EDMR spectra could only be observed above $250 \mathrm{~K}$ in accordance to EPR results. Surface and bulk transport could be separated and their analysis reflected the effect of magnetic interaction with oxygen. (C) 2007 American Institute of Physics.
\end{abstract}

[DOI: 10.1063/1.2719007]

\section{INTRODUCTION}

Conducting polymers have attracted increasing interest over the last decade due to the particular properties of the electronic states in a one-dimensional structure, as well as the near metallic conductivity that can be achieved upon doping. ${ }^{1}$ Polyaniline is one of the most studied conducting polymers due to its higher stability in air and its potential for commercial applications in various fields, such as electronics, electromagnetic shielding and sensors, among others. Its conductivity can be changed by protonation of the insulating emeraldine base form of polyaniline (EB-PANI).

Although all spins are paired in an ideal EB-PANI, even carefully synthesized samples yield an electron paramagnetic resonance (EPR) signal. The nature of these spins is still the subject of debate. ${ }^{2}$ Investigation of the magnetic susceptibility $(\chi)$ as a function of temperature $(T)$ often yields a Paulitype behavior ( $\chi$ independent of $T$ ), even for relatively low dc conducting polymers. ${ }^{3}$ However, in some cases, a transition from Curie to Pauli-type temperature dependence has been observed. ${ }^{3-5}$ Curie susceptibility is a characteristic signature of localized spins, while Pauli susceptibility is characteristic of delocalized spins. Why this transition is not always observed and how it relates to transport properties is still not completely clear.

Much progress has been made in the understanding of the conduction mechanism in different polymers; however, a detailed microscopic picture of the transport processes is not yet established for many of them. Such an investigation is an intricate subject. First, because of the very complex structure and morphology of conducting polymer films. Second, because only a few experimental techniques are capable of directly probing these processes selectively on the microscopic scale.

Electrically detected magnetic resonance (EDMR) measures the change in the sample conductivity under magnetic resonance condition. Thus it can relate microscopic properties to charge transport mechanisms, for example, identifying the electronic states taking part in the process. In fact, over the past years it has provided valuable insight into various transport and recombination processes in semiconductors. ${ }^{6-11}$

In this work we have used EDMR and EPR to investigate emeraldine base polyaniline films. We observed a transition of the magnetic susceptibility dependence on temperature, from a linear decrease with increasing temperature (localized Curie spins) to a constant value (delocalized Pauli spins). The $g$ factor also showed a transition at the same temperature $(240 \mathrm{~K})$. EDMR spectra could not be observed below $250 \mathrm{~K}$ in agreement with EPR results. The investigation of both surface and bulk transport yielded different spectral features that were explained by the influence of oxygen and water molecules.

\section{EXPERIMENT}

Deprotonated EB-PANI was obtained commercially. Films were prepared by casting a $0.1 \mathrm{M}$ N-methyl-2pyrrolidone (NMP) solution on glass and heating it at $60{ }^{\circ} \mathrm{C}$ until full evaporation of the solvent. At room temperature, films were immersed in milli-Q water and floated off the substrate. After drying we obtained flexible, free-standing films with typically a $20 \mu \mathrm{m}$ thickness. For EDMR, samples were cut to approximately $4 \times 8 \mathrm{~mm}^{2}$, and either coplanar (0.5 mm spacing) or sandwich silver contacts were deposited (with a contact area of $0.2 \mathrm{~cm}^{2}$ ).

EPR and EDMR measurements were done using a modified, computer interfaced, Varian E-4 X-band spectrometer. The temperature was varied using a $\mathrm{LN}_{2}$ flow cryostat, typically between 168 and $300 \mathrm{~K}$. At each step, we allowed the temperature to stabilize for at least $30 \mathrm{~min}$. The conductivity spin-dependent changes were measured by modulating the static magnetic field $\left(H_{0}\right)$ and using lock-in detection of the 

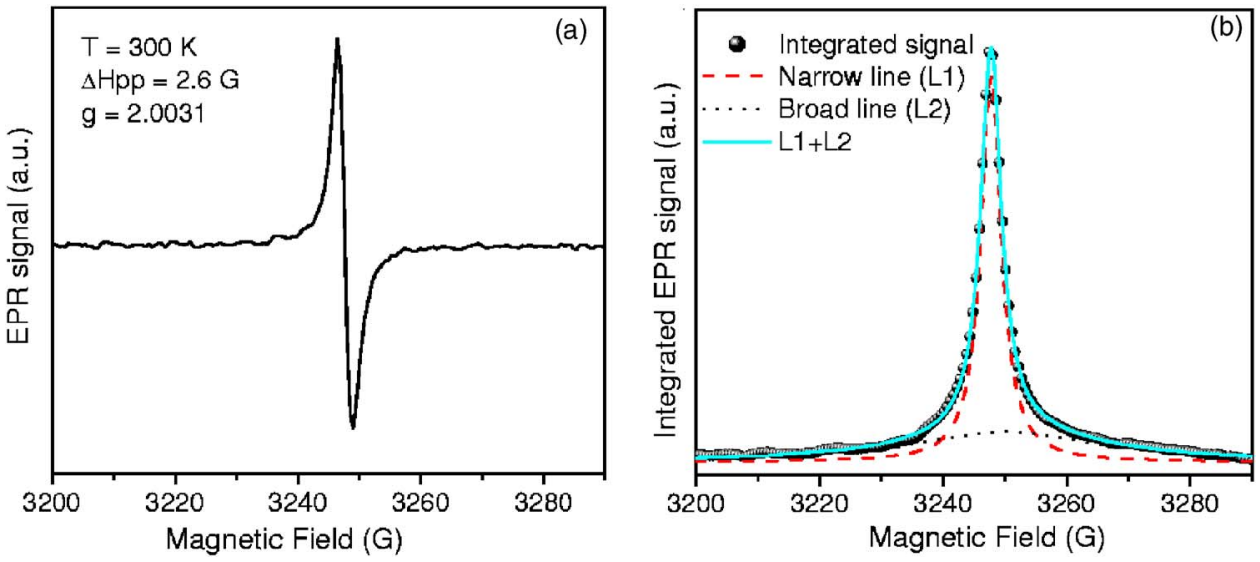

FIG. 1. (Color online) (a) EPR signal of a polyaniline film at room temperature; (b) Integrated EPR signal (solid spheres) decomposed into two Lorentzian lines, a narrow (dashed line) and a broad (dotted line) one. The solid line represents the sum of the two components $(\mathrm{L} 1+\mathrm{L} 2)$. resonant current changes. Magnetic susceptibility data were obtained from EPR spectra as being proportional to the signal intensity (the area under the absorption curve). DPPH was used as the standard to calibrate the measurements of the $g$ factor.

\section{RESULTS}

\section{A. Electron paramagnetic resonance}

Figure 1(a) shows the EPR signal of an undoped EB-PANI film at $300 \mathrm{~K}$. The peak-to-peak linewidth $\left(\Delta H_{p p}\right)$ is $2.6 \mathrm{G}$ and the $g$ factor is 2.0031 . These values are in agreement with those found in the literature, although the reported values may vary from ${ }^{4} 0.29$ to $23 \mathrm{G}^{12}$ These differences are due to magnetic interaction of polarons and the triplet state of oxygen molecules, ${ }^{13}$ which broadens the resonance line.

Fitting the EPR signals, we observed that it is composed of two Lorentzian lines: a narrow one, about $\Delta H_{p p}=2.3 \mathrm{G}$, and a large one, about $\Delta H_{p p}=17-23 \mathrm{G}$, depending on temperature [Fig. 1(b)]. It should be noted that it is not possible to simulate our signals using only one Lorentzian line.

At all measured temperatures, from 168 to $300 \mathrm{~K}$, the EPR spectra were always composed of two lines with more or less equal intensities. Polyaniline EPR spectra composed of two lines have already been reported. ${ }^{14,15}$ Mizoguchi et al. observed a single narrow Lorentzian for undoped samples, but as they raised the doping level, a second Lorentzian started to appear. Under very high doping levels this second component can become dominant. The appearance of this second component was also observed in an iodine doped polymer from the PPV family. ${ }^{3}$ In that work, the magnetic susceptibility dependence on temperature is very similar to that observed in our polyaniline samples, as will be discussed further in this paper.

The temperature dependence of the EPR signal is dominated by the narrow component. Therefore, the line shape analysis will be carried out only for this component. If we observe the temperature dependence of the magnetic susceptibility $(\chi)$, which is proportional to the area under the integrated EPR spectra, shown in Fig. 2, we can clearly see a transition behavior at approximately $240 \mathrm{~K}$.

The solid line, used to guide the eyes, shows that $\chi$ depends on the inverse of the temperature $(1 / T)$ between 168 and $233 \mathrm{~K}$, following Curie's Law. Then, there is a sudden increase and the value of $\chi$ becomes temperature independent between 258 and $300 \mathrm{~K}$, showing a Pauli-type behavior. Note that between 233 and $248 \mathrm{~K}$ the susceptibility dependence on temperature deviates from $1 / T$. The possible reason for this behavior will be discussed further on. Such a CuriePauli transition has already been observed in polyaniline ${ }^{4}$ and in other conducting polymers, ${ }^{3,5}$ such as polypirrol and polythiophene. However, the temperature $\left(T_{c}\right)$, at which this transition occurs, varies as a function of the sample's doping and crystallinity level. ${ }^{16,17}$

The peculiar behavior of the temperature dependence of the dominant's line $g$ factor, shown in Fig. 3, confirms that an important change occurs at this critical temperature. The $g$ factor value decreases from 2.0031 to 2.0028 as we raise the temperature from 168 to $228 \mathrm{~K}$. Then, there is a sudden increase to 2.0031 and $g$ remains practically temperature independent between 233 and $300 \mathrm{~K}$. The reason for this behavior is not completely understood at the moment; however, there is a clear relation to the Curie-Pauli transition.

The temperature dependence of the dominant component's linewidth is shown in Fig. 4(a). The solid line is used to guide the eyes. We observe that $\Delta H_{p p}$ decreases as tem-

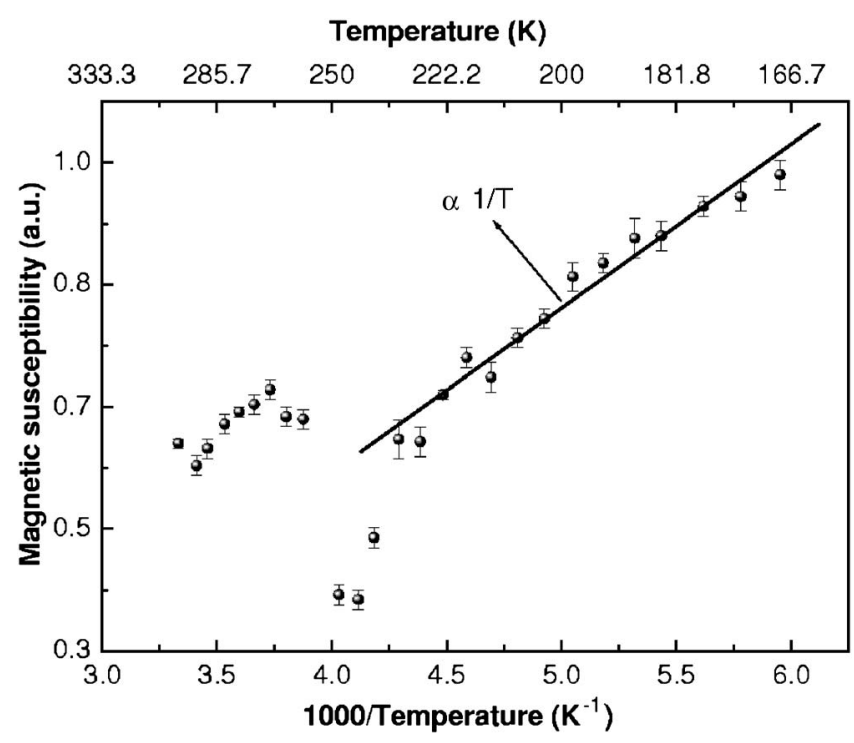

FIG. 2. Magnetic susceptibility temperature dependence for a PANI thin film. 


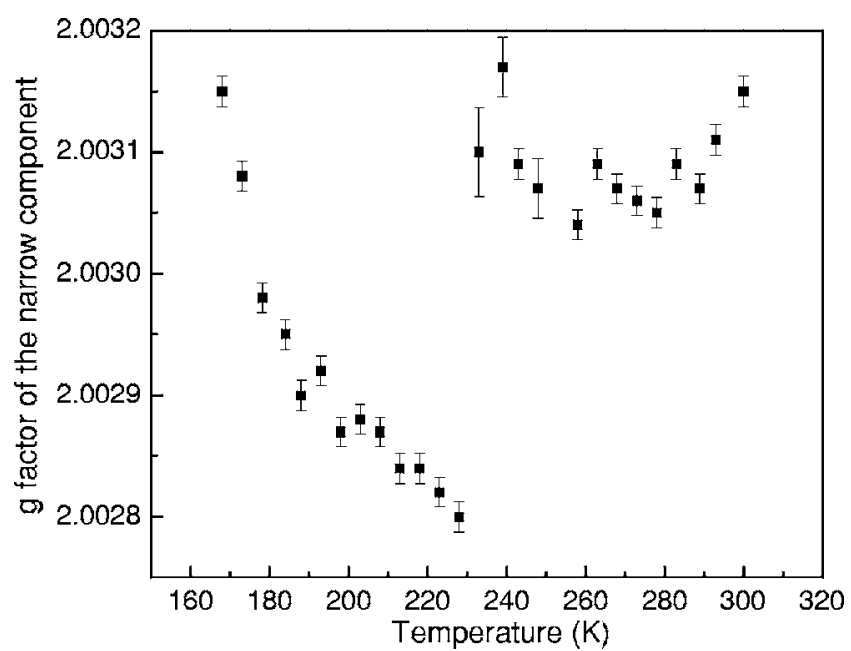

FIG. 3. Dependence of the thin component $g$ factor on temperature.

perature is increased. In general, EPR linewidths are determined by the interplay of spin-spin interactions (hyperfine, dipolar, etc.), spin-lattice relaxation and narrowing mechanisms (spin diffusion, rotation, and exchange).

In the case of conducting polymers, the hyperfine and dipolar broadening of a resonance will be limited by narrowing due to spin diffusion. ${ }^{18}$ An increase in conductivity, as observed in Fig. 4(b), will increase the effectiveness of spin diffusion to narrow the EPR linewidth.
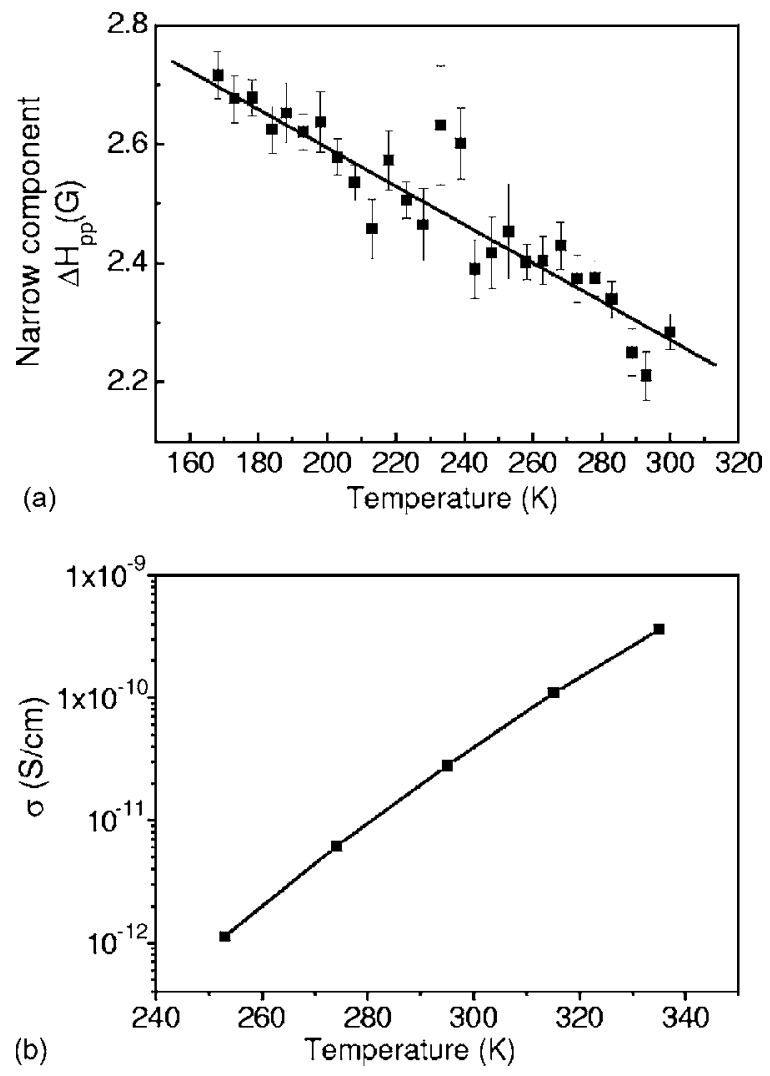

FIG. 4. Temperature dependence of: (a) the peak-to-peak linewidth of the dominant EPR component; (b) EB-PANI conductivity at $10^{4} \mathrm{~V} / \mathrm{cm}$.
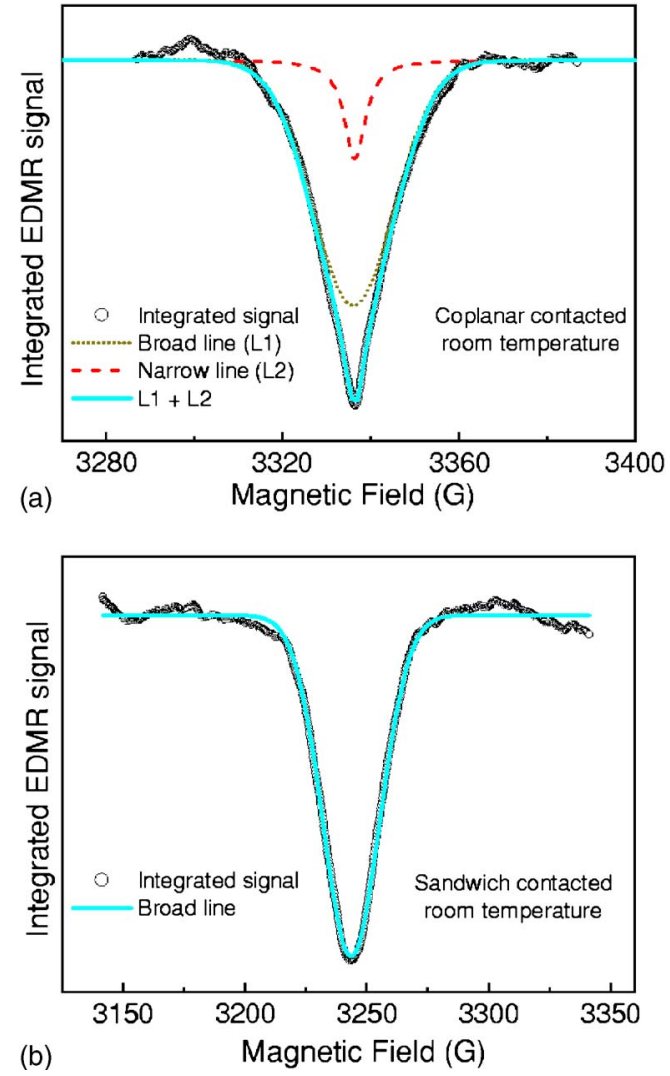

FIG. 5. (Color online) Integrated EDMR signal of: (a) A coplanar contacted device (open circles), decomposed into two components: a broad Gaussiantype (dotted line) and a narrow Lorentzian-type (dashed line). The solid line represents the sum of the two components $(\mathrm{L} 1+\mathrm{L} 2)$; (b) a sandwich type device (open circles) fitted using one broad Gaussian line (solid line).

\section{B. Electrically detected magnetic resonance}

No EDMR signal could be observed at temperatures below $250 \mathrm{~K}$. Different from the EPR results, the EDMR signal from polyaniline devices is dominated by a broad line. The signal is enhancing, meaning that conductivity increases during resonance, as would be expected for a hopping conduction. ${ }^{6,11}$ Two processes could result in this EDMR signal: interchain or intrachain polarons hopping. Note that in order to obtain an EDMR signal it is necessary that two spins participate in the observed process. One polaron will contribute to the signal when it hops to a site already occupied by another polaron. The resonance condition will enhance conductivity because it increases the spin dependent transition (hopping) rate.

Figure 5(a) shows the integrated EDMR signal (open circles) of a coplanar device, at $294 \mathrm{~K}$ and $50 \mathrm{~V}$ (electric field of the order of $10^{3} \mathrm{~V} / \mathrm{cm}$ ). The signal is also composed of two lines: a broad and a narrow line. The solid line represents the sum of the two components.

The narrow component has a $g$ factor of 2.0033 and $\Delta H_{p p}=3.2 \mathrm{G}$. This value is very close to that of the narrow component of the EPR signal. The broad component, on the other hand, has a $g$ factor slightly different, of 2.0035 and $\Delta H_{p p}=14.5 \mathrm{G}$. Graeff et $a l^{7}$ have observed a strong dependence of the narrow line intensity with the applied voltage. According to the authors, the narrow component is dominant at low electric fields $\left(<10^{3} \mathrm{~V} / \mathrm{cm}\right)$ and strongly decreases as 
the field is increased. The appearance of a broad component was only observed for fields above $10^{3} \mathrm{~V} / \mathrm{cm}$.

The EDMR signal from sandwich devices is composed of only one broad line with $g$ factor of 2.0035 and $\Delta H_{p p}=22 \mathrm{G}$. The integrated signal (open circles) and the fitted line (solid line) can be seen in Fig. 5(b). Possible reasons for the differences observed between coplanar and sandwich devices will be discussed further on.

\section{DISCUSSION}

EPR measurements on polyaniline films have shown a transition in the spin system from Curie-type to Pauli-type, as we raise the temperature. Below $\sim 240 \mathrm{~K}$, magnetic susceptibility decreases with increasing temperature and above this temperature it is independent of $T$. Curie spins are localized, therefore, they do not contribute to the current, and, as a consequence, to the EDMR signal. On the other hand, the Pauli-type contribution comes from delocalized spins, which do contribute to the current. ${ }^{19}$ That could explain why no EDMR signal is observed below $250 \mathrm{~K}$ for these polyaniline samples.

Numerical calculations ${ }^{17,20}$ have shown that at sufficiently low temperatures, so that $k_{B} T$, where $k_{B}$ is Boltzmann constant, is smaller than the average Coulomb interaction energy $(U)$ among electrons, states near the Fermi energy $\left(E_{F}\right)$ will become singly occupied, and magnetic susceptibility will exhibit a Curie behavior

$$
\chi(T)=\mu_{B}^{2} N_{S} / k T,
$$

where $N_{S}$ is the number of singly occupied states per volume unit. Considering a low density of states at the Fermi energy, the Curie contribution at low temperatures comes from the occupancy of localized states close to $E_{F}$.

When $E_{F}$ is close to the mobility edge and much higher than the interaction energy $U$, the spin susceptibility dependence on temperature will gradually change from a Curietype to a Pauli-type contribution, as we raise $T$. The transition region occurs when $U \approx$ thermal energy.

Since crystallinity and doping levels can alter the Fermi energy and/or electron-electron interaction energy, different research groups have (or have not) observed the transition present in Fig. 2 depending on doping and/or the crystallinity level of the studied samples. ${ }^{4,16}$ The transition temperature is higher where there is higher disorder and/or at lower doping levels. As most of the EPR studies on polyaniline are carried out with the polymer in powder, many research groups only observed the Curie-Pauli transitions in highly doped samples. ${ }^{14}$ In those cases, they observed that the signal was composed of two Lorentzian lines, a broad and a narrow one, in agreement with our results. However, in Ref. 14 the broad component was dominant, while in our case it is not. According to Mizoguchi et al., ${ }^{14}$ the broader component is not present in EPR spectra of undoped polyaniline samples, but starts to appear as the doping level is raised, becoming dominant at high doping levels. One interesting question is the origin of such behavior.

It is believed that $\mathrm{H}_{2} \mathrm{O}$ molecules are adsorbed preferentially by localized spin sites in polyaniline. ${ }^{12}$ Since the ad- sorption sites, where the polaron is centered (i.e., $\mathrm{NH}^{+}$), are the same for $\mathrm{O}_{2}$ and $\mathrm{H}_{2} \mathrm{O}$ molecules, the higher water adsorption leads to less free sites for oxygen to be adsorbed. Houzé and Nechtschein ${ }^{13}$ proposed that $\mathrm{H}_{2} \mathrm{O}$ molecules are more tightly bound to the polymeric polyaniline chain than $\mathrm{O}_{2}$ molecules, and that water acts as a shield between polarons and oxygen. Thus, as the doping level is increased, we have more delocalized polarons, and, consequently, more sites for oxygen adsorption, which will broaden the EPR signal due to dipole-dipole interactions with paramagnetic species. The fact that the narrow component is dominant in our spectra comes from the low (unintentional) doping level of our samples.

Peak-to-peak linewidths $\left(\Delta H_{p p}\right)$, equal to ${ }^{12} 2.2$ and 2.0 $\mathrm{G},{ }^{13}$ have been reported, respectively, to samples in the water vapor atmosphere and ambient atmosphere. These results are very close to the values of $\Delta H_{p p}$ obtained in this work $\left(\Delta H_{p p}=2.6 \mathrm{G}\right)$, in which the films were prepared and kept in air. The fact that we observe two components, a narrow and a broad one, represents the contribution of both localized (sites mostly occupied by $\mathrm{H}_{2} \mathrm{O}$ ) and delocalized (sites mostly occupied by $\mathrm{O}_{2}$ ) polarons. ${ }^{21}$

In EDMR measurements, the broad signal is dominant since it is due, mostly, to delocalized polarons. However, at low bias, for coplanar contacted devices, we observe only a narrow component of decreasing intensity as bias is raised. ${ }^{7}$ The narrow component, in analogy to EPR, ${ }^{12,13}$ is attributed to polarons near $\mathrm{H}_{2} \mathrm{O}$, meaning, in this case, polarons close to the surface. Near the surface, water molecules have a higher probability of being adsorbed, lowering the effect of oxygen broadening. As bias is raised, the electric field starts to further penetrate into the bulk, where there is no longer the competition between $\mathrm{H}_{2} \mathrm{O}$ and $\mathrm{O}_{2}$ molecules. At a higher bias, the bulk component increases relative to the surface component, and the intensity of the narrow line decreases. This same idea can be applied to EPR. The samples in powder form have a much higher surface area relative to films. Thus, as observed, ${ }^{14}$ EPR signals in this case are dominated by one narrow Lorentzian line.

\section{Curie-Pauli transition}

Mizoguchi ${ }^{16}$ and Wang et al. ${ }^{19}$ have shown that macroscopic conductivity is dominated by interchain and/or intermetallic island hopping. According to Krinichnyi, ${ }^{22}$ macroscopic conductivity in polyaniline, both ac and $\mathrm{dc}$, is thermally activated at approximately $200 \mathrm{~K}$. Therefore, we have attributed our EPR signals, at high temperatures, to movable polarons thermally activated above approximately $220 \mathrm{~K}$. The EDMR signal is attributed to interchain hopping. The formation of bipolaron states is energetically favorable relative to the polaronic formation. ${ }^{3}$ Thus bipolaron formation increases with decreasing temperature, leading to smaller magnetic susceptibility values. When the polaron density is small enough, the fusion (bipolaron formation) process no longer takes place and the Curie behavior at low temperatures is observed due to the fixed number of localized polarons. This also explains why the susceptibility deviates from $T^{-1}$ dependence, between 233 and $248 \mathrm{~K}$. 


\section{CONCLUSION}

We used electron paramagnetic resonance and electrically detected magnetic resonance to investigate polyaniline films. We observed a Curie-Pauli spin transition as a function of temperature that is reflected in an abrupt change of the $g$ factor. The EPR linewidth decreased linearly with an increasing temperature due to an enhanced spin diffusion. The study of coplanar and sandwich type devices highlighted different EDMR signals for surface and bulk currents. We concluded that EPR signals are also composed of a surface and a bulk contribution. The competition between oxygen and water adsorption on the surface could account for the differences observed. Due to the strong influence of oxygen on the linewidth, we propose that EDMR can access encapsulations. Any oxygen induced broadening would be readily detected and, different from EPR, no signal from the materials used for encapsulation would be observed.

\section{ACKNOWLEDGMENTS}

The authors are pleased to acknowledge financial support from the following Brazilian agencies: FAPESP and CNPq (IMMP).

${ }^{1}$ A. G. MacDiarmid and A. J. Epstein, in Science and Applications of Conducting Polymers, edited by W. R. Salaneck, D. T. Clark, and E. J. Samuelson (Hilger, Bristol, 1991), p. 117.

${ }^{2}$ P. K. Kahol, A. Raghunathan, and B. J. McCormick, Synth. Met. 140, 261 (2004).
${ }^{3}$ C. E. Lee, D. K. Oh, J. L. Jin, and B. K. Nam, Synth. Met. 69, 425 (1995).

${ }^{4}$ N. S. Sariciftci, A. J. Heeger, and Y. Cao, Phys. Rev. B 49, 5988 (1994).

${ }^{5}$ H. S. Nalwa, Phys. Rev. B 39, 5964 (1989).

${ }^{6}$ M. Stutzmann, M. S. Brandt, and M. W. Bayerl, J. Non-Cryst. Solids 266-269, 1 (2000).

${ }^{7}$ C. F. O. Graeff, M. S. Brandt, R. M. Faria, and G. Leising, Phys. Status Solidi A 162, 713 (1997).

${ }^{8}$ I. Hiromitsu, Y. Kaimori, M. Kitano, and T. Ito, Phys. Rev. B 59, 2151 (1999).

${ }^{9}$ F. A. Castro, G. B. Silva, L. F. Santos, R. M. Faria, F. Nüiesch, L. Zuppiroli, and C. F. O. Graeff, J. Non-Cryst. Solids 338-340, 622 (2004).

${ }^{10}$ F. A. Castro, G. B. Silva, F. Nüesch, L. Zuppiroli, and C. F. O. Graeff, Org. Electron. 8, 249 (2007)

${ }^{11}$ C. F. O. Graeff, G. B. Silva, F. Nüesch, and L. Zuppiroli, Eur. Phys. J. E 18, 21 (2005).

${ }^{12}$ P. K. Kahol, A. J. Dyakonov, and B. J. McCormick, Synth. Met. 84, 691 (1997).

${ }^{13}$ E. Houze and M. Nechtschein, Phys. Rev. B 53, 14309 (1996).

${ }^{14}$ K. Mizoguchi, T. Obana, S. Ueno, and K. Kume, Synth. Met. 55, 601 (1993).

${ }^{15}$ A. V. Kulikov, V. R. Bogatyrenko, O. V. Belonogova, L. S. Fokeeva, A. V. Lebedev, T. A. Echmaeva, and I. G. Shunina, Russ. Chem. Bull. 51, 2216 (2002).

${ }^{16}$ K. Mizoguchi, Jpn. J. Appl. Phys., Part 1 34, 1 (1995).

${ }^{17}$ M. N. Bussac and L. Zuppiroli, Phys. Rev. B 47, 5493 (1993).

${ }^{18}$ B. R. Weinberger, E. Ehrenfreund, A. Pron, A. J. Heeger, and A. G. MacDiarmid, J. Chem. Phys. 72, 4749 (1980).

${ }^{19}$ Z. H. Wang, E. M. Scherr, A. G. MacDiarmid, and A. J. Epstein, Phys. Rev. B 45, 4190 (1992).

${ }^{20}$ H. Kamimura, Philos. Mag. B 42, 763 (1980); in Electron-Electron Interactions in Disordered Systems, edited by A. L. Efros and M. Polak (NorthHolland, Amsterdam, Netherlands, 1985).

${ }^{21}$ V. Sitaram, A. Sharma, S. V. Bhat, K. Mizoguchi, and R. Menon, Phys. Rev. B 72, 035209 (2005).

${ }^{22}$ V. I. Krinichnyi, Synth. Met. 108, 173 (2000). 